\title{
MASTER
}

\section{ON THE STRUCTURAL BEHAVIOR OF PROGRESSIVELY MINED SOLUTION CAVITIES IN SALT}

\author{
A. F. Fossum
}

March 1977

This paper was prepared for journal publication by RE/SPEC Inc. under the provisions of Consultant Subcontract 4269 and Purchase Order Subcontract 89Y-22303C with Union Carbide Corporation, Nuclear Division. The subcontract was administered by Oak Ridge National Laboratory and the Office of Waste Isolation. OAK RIDGE. TENNESSEE 


\section{DISCLAIMER}

This report was prepared as an account of work sponsored by an agency of the United States Government. Neither the United States Government nor any agency Thereof, nor any of their employees, makes any warranty, express or implied, or assumes any legal liability or responsibility for the accuracy, completeness, or usefulness of any information, apparatus, product, or process disclosed, or represents that its use would not infringe privately owned rights. Reference herein to any specific commercial product, process, or service by trade name, trademark, manufacturer, or otherwise does not necessarily constitute or imply its endorsement, recommendation, or favoring by the United States Government or any agency thereof. The views and opinions of authors expressed herein do not necessarily state or reflect those of the United States Government or any agency thereof. 


\section{DISCLAIMER}

Portions of this document may be illegible in electronic image products. Images are produced from the best available original document. 
This report was prepared as an account of work sponsored by the United States Government. Neither the United States nor the Energy Research and Development Administration, nor any of their employees, nor any of their contractors, subcontractors, or their employees, makes any warranty, express or implied, or assumes any legal liability or responsibility for the accuracy, completeness or usefulness of any information, apparatus, product or process disclosed, or represents that its use would not infringe privately owned rights. 
ON THE STRUCTURAL BEHAVIOR OF PROGRESSIVELY

HINED SOLUTION CAVITIES IN SALT

\author{
by \\ A. F. Fossum \\ Resident Research Engineer \\ RE/SPEC Inc. \\ Rapid City, SD 57701
}

\begin{abstract}
.
The stress field and the time dependent deformation around a solution cavity in rock salt are shown to be sensitive to the details of the excavation. process and to the prevailing tectonic stress field. An analysis procedure is developed whereby an incremental excavation procedure and a creep routine are incorporated simultaneously into an axisymmetric finite element code.
\end{abstract}

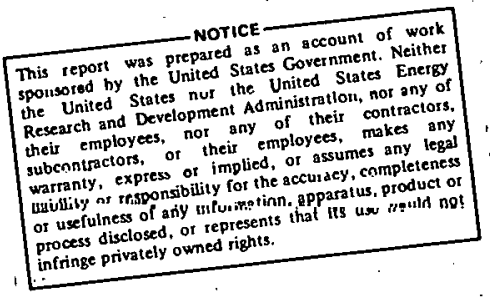


1. INTRODUCTION

Previous papers $[1,2,3,4]$ concerned with the creep behavior of cavities in salt domes or massive salt bodies included the assumption that the cavities were created instantaneously. In point of fact, the evolution of a cavity may take a year or more to develop, the evolving shape of the cavity changing continually with time.

In this paper a procedure will be adopted specifically for the time dependent analysis of a progressively mined cavity. The procedure will allow the cavity to be constructed in increments to its final configuration consistent with the intermediate shapes and excavation times observed in the field. In rock mechanics applications it is important that the analysis procedure accommodate a rather arbitrary initial stress field. This is evident in the present application since although every salt dome is subjected to forces due to the weight of the overburden, not all of them have the same horizontal compression. In some areas tectonic movements cause horizontal stresses which can be quite different than those resulting in a purely gravitating medium. Gera [5] has discussed some of the aspects of salt tectonics in salt formations used for the disposal of radioactive wastes and gives an extensive list of references.

It is not the purpose of this paper to derive a creep law or to discuss the merits or inadequacies of creep laws used in previous analyses. But rather, a constitutive law which has been used by other authors for the analysis of salt cavities is used to demonstrate that the structural behavion in the vicinity of the cavity is sensitive to the details of the excavation process and to the prevailing tectonic stress state. The creep law is taken from Nair et al. [1] and is a power law in time and stress level given by 


$$
\left\{\varepsilon^{c}\right\}=1.87\left(10^{-13}\right) t^{0.36}\left(/ 3 \mathrm{~J}_{2}^{1 / 2}\right)^{2.98}(3 / 2)\{s\} /\left(/ 3 \mathrm{~J}_{2}^{1 / 2}\right)
$$

with the stress deviator $\{s\}$ and the second invariant of stress deviator, $\mathrm{J}_{2}$. It is converient to use the notation implied by equation ( 1 ) in order to discuss the numerical algorithm. In this notation vectors are enclosed by (1) and matrices by []. Thus in equation (1), the creep strain is written as $\left\{\varepsilon^{c}\right\}^{T}=\left[\varepsilon_{x}^{c}, \varepsilon_{y}^{c}, \varepsilon_{z}^{c}, \varepsilon_{x y}^{c}, \varepsilon_{y z}^{c}, \varepsilon_{z x}^{c}\right]$ and similarly for the stress deviator $\{s\}$.

\section{NUMERICAL ALGORITHM}

$i$

In this section an algorithm is adopted which includes the simultaneous construction and creep of a cavity. This procedure is similar to that of Zienkiewicz et al. [6] and the interested reader is referred to this paper and to one by Comeau [7] in which the numerical stability problem is discussed for this solution technique. The principle of virtual work, leads one to the repeated solution of a modified elastic problem involving time dependent displacements $\{\delta\}$ given by

$$
[K]\{\delta\}-\int_{\Omega}[B]^{T}[D]\left\{\varepsilon^{c}\right\} d \Omega-\left\{F^{*}\right\}=\{0\}
$$

in which $[K]$ is the standard stiffness matrix of the structure

$$
[K]=\int_{\Omega}[B]^{T}[D][B] d \Omega,
$$

[B] is the strain-displacement matrix, [D] is the matrix of elastic properties, $\left\{\varepsilon^{c}\right\}$ is the creep strain and.

$$
\left\{F^{*}(t)\right\}=\{F\}+\int_{\Omega}[B]^{T}[D]\left\{\varepsilon^{0}\right\} d \Omega-\int_{\Omega}[B]^{T}\left\{\sigma_{0}\right\} d \Omega
$$


is the force vector which includes body and boundary loads, and loads due to initial stresses $\left\{\sigma_{0}\right\}$ present at the start of the problem, and to initial strains $\left\{\varepsilon^{0}\right\}$. The stress vector is expressed as

$$
\{\sigma\}=[D][B]\{\delta\}-[D]\left\{\varepsilon^{c}\right\}+\left\{\sigma_{0}\right\}-[D]\left\{\varepsilon^{0}\right\}
$$

In the final section it will be shown that creep behavior in the vicinity of the cavity is affected significantly by tectonic stresses and hence a discussion of the initial stresses $\left\{\sigma_{0}\right\}$ is in order. It is assumed at the start of the problem that a self equilibrating initial stress field is superposed onto a gravitating stress field. The self equilibrating initial stress field, the tectonic stresses, are assembled into the initial stress vector $\left\{\sigma_{0}\right\}$. Note that since the initial stress vector $\left\{\sigma_{0}\right\}$ is now self equilibrating, the forces,

$$
\{F\}_{\sigma_{0}}=\int_{\Omega}[B]^{T}\left\{\sigma_{0}\right\} d \Omega
$$

are identically zero after assembly. After an excavation a force imbalance will result from equation (6). If the gravitating stress field plus tectonic stresses are desired to exist at zero displacement at the start of the problem, equation (4) must be identically equal to zero provided no inelastic strains exist at time $t=0$. Thus from equation (4),

$$
\{F\}=-\int_{\Omega}[B]^{T}[D]\left\{\varepsilon^{0}\right\} d \Omega .
$$

That is, the forces due to initial strains exactly balance the body and surface forces. 
A simplification in this procedure is possible if the last two terms in equation (5) are combined in the following manner. The vertical stresses in a gravitating medium are assumed to be given by $\sigma_{z z}=\rho g z$ where $\rho$ is the mass density, $g$ is the gravitational constant and $z$ is measured from the surface so that compression is negative. The horizontal stresses are assumed to be given by a combination of gravitating and tectonic stresses in the form

$$
\left[\sigma_{x x}, \sigma_{y y}, \sigma_{z z}, \sigma_{x y}, \sigma_{y z}, \sigma_{z x}\right]=\left[K_{p g z}, K_{p g z}, p g z, 0,0,0\right]
$$

where $K$ is arbitrary. Note that if $K$ is given by $v /(1-v)$, where $v$ is Poisson's ratio, a gravitating stress field results in which there are no horizontal displacements anywhere. If $\mathrm{K}=1$, a lithostatic stress field results. The possibility also exists that $K$ could be greater than one, in which case the horizontal stresses would be greater than the vertical stresses. This last case is probable for a salt dome which is being "squeezed" upward (diapiric process). At the start of the problen an initial stress $\left\{\sigma^{0}\right\}$ defined by

$$
\left\{\sigma^{0}\right\}=\left\{\sigma_{0}\right\}-[D]\left\{\varepsilon^{0}\right\}
$$

is constructed according to equation ( 8 ). Thus an initial elastic run is not necessary since, initially, equation (5) is given by equation (9). In the incremental solution procedure, the total current stresses $\left\{\sigma_{i}\right\}$ will be given by

$$
\left\{\sigma_{i}\right\}=\left\{\sigma^{0}\right\}+\sum_{i}\left\{\Delta \sigma_{i}\right\}
$$

where $\left\{\Delta \sigma_{i}\right\}$ is calculated for each time step or excavation increment. 
The creep strains and displacements are assembled similarly with time. The solution technique consists of the following sequence:

(i) The creep strain change is estimated from the total current stress level $\left\{\sigma_{i}\right\}$ over a time interval $\Delta t$ by

$$
\left\{\Delta \varepsilon^{c}\right\}=\int_{t}^{t+\Delta t}\left\{\dot{\varepsilon}^{c}\right\} d t
$$

(ii) The total current inelastic strains are updated according to

$$
\left\{\varepsilon_{t+\Delta t}^{c}\right\}=\left\{\varepsilon_{t}^{c}\right\}+\left\{\Delta \varepsilon^{c}\right\}
$$

(iii) The total current displacements are updated according to

$$
\left\{\delta_{t+\Delta t}\right\}=\left\{\delta_{t}\right\}+\{\Delta \delta\}
$$

where $\{\Delta \delta\}$ is calculated from

$$
[K]\{\Delta \delta\}=\int_{\Omega}[B]^{T}[D]\left\{\Delta \varepsilon^{C}\right\} d \Omega
$$

(iv) The total current stresses are updated according to equation (10) where $\left\{\Delta \sigma_{i}\right\}$ is calculated from

$$
\left\{\Delta \sigma_{i}\right\}=[D][B]\{\Delta \delta\}-[D]\left\{\Delta \varepsilon^{c}\right\}
$$

The process is then repeated beginning from (i). The excavation procedure is assumed to occur (as an approximation to a field situation) as a sequence of instantaneous excavations. When the appropriate time is reached for one 
of these excavations the stiffness matrix $[K]$ is updated by the removal of elements and a consistent load vector $\{\Delta Q\}$ is formed corresponding to the static head of fluid and the reverse of the normal and shear stresses existing prior to excavation on the tangent plane to the new cavity surface. The increment in displacements $\{\Delta \delta\}$ is calculated from

$$
\left[K_{\text {new }}\right]\{\Delta \delta\}=\{\Delta Q\}
$$

and the increment in stresses is calculated from

$$
\{\Delta \sigma\}=[D][B]\{\Delta \delta\}
$$

and then added to the total current displacements $\{\delta\}$ and stresses $\{\sigma\}$ respectively. Since each excavation is instantaneous, it is assumed that the maximum stress level is reached accompanied only by elastic strains. Flow is then allowed to continue at the new stress level.

A number of theoretical aspects of the sequential construction and/or excavation procedure has been discussed in the literature. In particular, the reader is referred to the work by Brown and Goodman [8], Goodman and Brown [9], and Brown and King [10]. Physically, the process of excavation involves removing material from an already stressed structure. By the removal of material, a new stricture has been created which corresponds to a new stiffness matrix. The newly created boundary surface is characterized by the vanishing of normal and shear stresses on that boundary except in the case of the solution mined cavity in which a normal pressure is imposed on the surface corresponding to the static head of.fluid. Note that the stress state existing just before mining would remain unchanged only if the 
normal and shear stresses existing at the position in the pre-mining configuration corresponding to the newly created surface in the post-mining configuration were applied to the new configuration. In this context, normal and shear stresses refer to stresses directed normal and tangent respectively to the new surface.

The following procedure has been adopted as a means for convenient. reformulation of the stiffness matrices. The elements which are excavated first are numbered last. The elements which are excavated second are numbered second to last and so on. The assembly of the stiffness matrix proceeds element by element beginning with element 1. When the assembly reaches the element to be excavated last, this matrix is written on a direct access unit with zeros occupying the unfilled positions. The assembly then continues until the last element of the group of elements to be excapated second to last is reached and then this matrix is written on another direct access unit again with zeros occupying the unfilled positions. This procedure continues until the last element is reached. When the time to excavate is reached, the appropriate stiffness matrix is read into core. At this time, the positions of the stiffness matrix corresponding to the. diagonals are checked for zeros. When one occurs say at $K_{\ell l}$ it is replaced by unity. The equilibrium equation is additionally modified by placing a zero in the lth position of the load vector. This procedure prevents the stiffness matrix from becoming singular and simply results in zero displacements for the excavated nodes. The stresses at the excavated nodes are then set to zero. This procedure allows the nodal numbering scheme to remain unchanged.

At the time of excavation, the state of stress on the new cavity boundary is examined. If the boundary is skewed with respect to the global coordinates 
or if the boundary is curved, a tensor transformation is performed in order to obtain the normal and shear stresses existing on the new face. The use of isoparametric elements greatly facilitates this procedure. Not only can nodal stresses be obtained, but the spatial derivatives of the interpolation polynomial used previously to obtain the strain displacement matrices may be used to obtain the appropriate slope angles of the new cavity boundary.

\section{FINITE ELEMENT SOLUTION OF CREEP PROBLENS}

The examples considered in this section were selected to illustrate the influence of the excavation simulation on the azimuthal stress distribution around a cavity and the importance of the tectonic stress state on creep behavior. For simplicity, a spherical cavity has been chosen. The Finite element mesh of this cavity is illustrated in Figure 1. Shaded elements are shown in the interior of the spherical boundary which will be excavated in the sequence indicated by the numbers between the bold-faced boundaries. Once these elements have been excavated, the mesh will represent a spherical cavity in a half-space. Before excavation the mesh contains 233 biquadratic isoparametric ring elements with 760 nodes. After the final excavation the mesh contains 128 elements with 433 nodes.

The first example was chosen to illustrate the adequacy of the creep routine and the method of dealing with initial stresses. The example, one of several considered by Nair et al., [1] involves the instantaneous construction and creep of a spherical cavity in a lithostatic stress field. The nodes along $\mathbf{r}=0$ were rollered to prevent horizontal displacement as required by symmetry. Next a single excavation to the final configuration was made according to the procedure given in Section 2 . That is, incremental displacements and stresses were obtained from equations (16) and (17) and 
added to the total displacements $\{\delta\}$ and stresses $\{\sigma\}$. In this example the cavity has a diameter of 96 meters and is located at a depth of 823 meters. from the surface to the center of the cavity. The variation of vertical stress with depth is $0.0226 \mathrm{MPa} /$ meter. Young's modulus and Poisson's ratio are respectively $2.76\left(10^{4}\right) \mathrm{MPa}$ and 0.45 . The creep law is given by equation. (1). In Figure 2 the horizontal stress around the periphery of the cavity is shown immediately after the instantaneous excavation at $t=0$ and after 24 hours of creep. As illustrated in the figure, Nair's results differ only slightly with the present results. The results by Nair were obtained with constant strain elements and hence the horizontal stress is not zero at $90^{\circ}$ since the stresses were calculated at the centers of the elements. The figure also includes the results for the case in which the washing fluid pressure is imposed on the face of the cavity. The brine หas assumed to have a specific gravity of 1.1. The variation of the horizontal stresses around the cavity is considerably smoother in the presence of the fluid pressure.

The next example is chosen to illustrate the importance of the excavation sequence on the azimuthal stress distribution around the cavity. The excavation sequence is illustrated in the mesh of Figure 1 with the numbers representing the order in which the segments of elements are removed. This type of excavation might result from the bottom hole injection method of solution mining. The excavations occurred at times $t=0.25,0.5,0.7$, and 0.9 years. The creep law was sirilar to the one used by Nair et al. [1], and the initial stress state was lithostatic. Hith the final configuration as the reference configuration the horizontal and vertical azimuthal stress distributions are shown in Figres, 3 and 4 respectively. That is, the horizontal or vertical stress at the reference configuration after each 
excavation is plotted versus the azimuthal angle measured from the bottom of the cavity. The washing fluld pressure has been included in this analysis. The straight lines in Figures 3 and 4 represent the pre-mining state of stress which is higher at the botton than at the top of the cavity due to gravity. The distribution of horizontal stress for this case is quite different than the distribution of horizontal stress in the previous example. Not only is the distribution less symnetric but whereas in the previous example the maximum horizontal stress occurred at the bottom of the cavity, now it occurs at the top. This is due to the fact that during excavation, the stresses near the bottom of the cavity are relaxing before the upper part of the cavity is completed. The vertical stress distribution shows similar behavior with the exception that near the equator some extremely severe stress gradients are observed due to the sharp comers that exist after the thind excavation.

The final example involves an observation of the creep behavior under different initial stress conditions. The procedure is identical to that zused in the previous example with the execptinn that following the final rexcavation, the cavity is dewatered. That is, the fluid is evacuated. This step is necessary for example in the event that radioactive waste material sould be stored in the cavity. The values chosen for the lateral earth xpressure coefficient $K$ as defined earlier, were $2 / 3,1$, and $4 / 3$. This represents a very wide range of values as most salt domes in the U.S. will exhibit values of $K$ very close to 1. The dowmard, inward, and upward displacements at the top, side, and bottom of the cavity respectively are shown plotted versus time in Figures 5,6, and 7. These figures demonstrate the extreme sensitivity of creep to the prevailing initial stress field. Note that for the case in which $K=4 / 3$, the entire cavity appears to rise. It 
should be evident from these figures that even slight deviations from a lithostatic situation would result in significant differences in long term creep displacements. Long term in this context may be of the order of one hundred years or more. Thus an accurate assessment of the initial in situ stress state is practically a necessity.

Finally, it should be noted that in this study, the fluid was evacuated instantaneously resulting in the sudden jumps in displacement at approximately one year in Figures 5,6 , and 7 . The excavation sequence used here is purely hypothetical and no attempt was made at modeling a precise field situation which may involve different intermediate configurations and excavation times.

\section{ACKNOWLEDGMENTS}

The Financial support provided by the Oak Ridge National Laboratory (Union Carbide Corporation, Nuclear Division) under Subcontract No. 4269 for portions of this work is gratefully. acknowledged.

\section{REFERENCES}

1. Nair, K., Sandhu, R. S., and Wilson, E. L., "Time-Dependent Analysis of Underground Cavities under Arbitrary Initial Stress Field," Proc. 10th Symp. on Rock Mech., Austin, Texas, 1968.

2. Anderson, C.A., "An Investigation of the Steady Creep of a Spherical Cavity in a Half Space," Journal of Applied Mechanics, Vol. 43, Trans. ASME, Vol. 90, Series E, 1976, P. 254 .

3. Boresi, A. P. and Deere, D. U., "Creep Closure of a Spherical Cavity in an Infinite Medium," Einal report to Holmes $\varepsilon$ Narver, Inc. Las Vegas Division, May, 1963. 
4. Hinkel, B. V., Gerstle, K. H., and Ko, H. Y., "Analysis of TimeDependent Deformations of Openings in Salt Media," Int. J. Rock Mech. Min. Sci. Vol. 9, 1972, p. 249.

5. Gera, F., "Review of Salt Tectonics in Relation to the Disposal of Radioactive Wastes in Salt Fomations," Geol. Soc. Amer. Bull. Vol. 83, 1972, p. 3551.

6. Zienkiewicz, O. C. and Cormeau, I. C., "Visco-Plasticity--Plasticity and creep in Elastic Solids--A unified Numerical Solution Approach," Int. Jour. for Numerical Methods in Eng., Vol. 8, 1974, p. 821. i 7. Cormeau, I. C., "Numerical Stability in Quasi Static Elastovisco-plasticity," Int. Jour. for Numerical Methods in Eng., Vol. 9, 1975. 8. Brown, C. B., and Goodman; L. E., "Gravitational Stresses in Accreted Bodies," Proceedings Series A. Vol. 276, Royal Society, London, England, 1963, p. 571.

9. Goodman, L. E., and Brown, C. B., "Dead Load Stresses and the Instability of Slopes," Journal of the Soil Mechanics and Foundations Division, ASCE, Vol. 89, No. SM3, Proc. Paper 3514, May, 1963, p. 103. 10. Brown, C. B. and King, I. P., "Automatic Embankment Analysis: Equilibrium and Instability Conditions," Geotechnique, Vol. 16, No. 3, Institution of Civil Engineers, London, England, 1966, p. 209. 


\section{LIST OF FIGURE CAPTIONS}

FIGURE \& Finite Element Mesh of a Spherical Cavity with Elements to be Excavated Shown Shaded

FIGURE 2 Horizontal Stress around a Spherical Cavity

FIGURE $3^{\circ}$ Influence of Excavation Sequence on Horizontal Stress around a Cavity

EIGURE 4 Influence of Excavation Sequence on Vertical Stress around a Cavity

EIGURE 5 Influence of Tectonic Stresses on the Creep Behavior of a Cavity (Downward Displacement)

FIQURE 6 Influence of Tectonic Stresses on the Creep Behavior of a Cavity (Inward Displacement)

FIGURE 7 Influence of Tectonic Stresses on the Creep Behavior of a Cavity (Upward Displacement) 


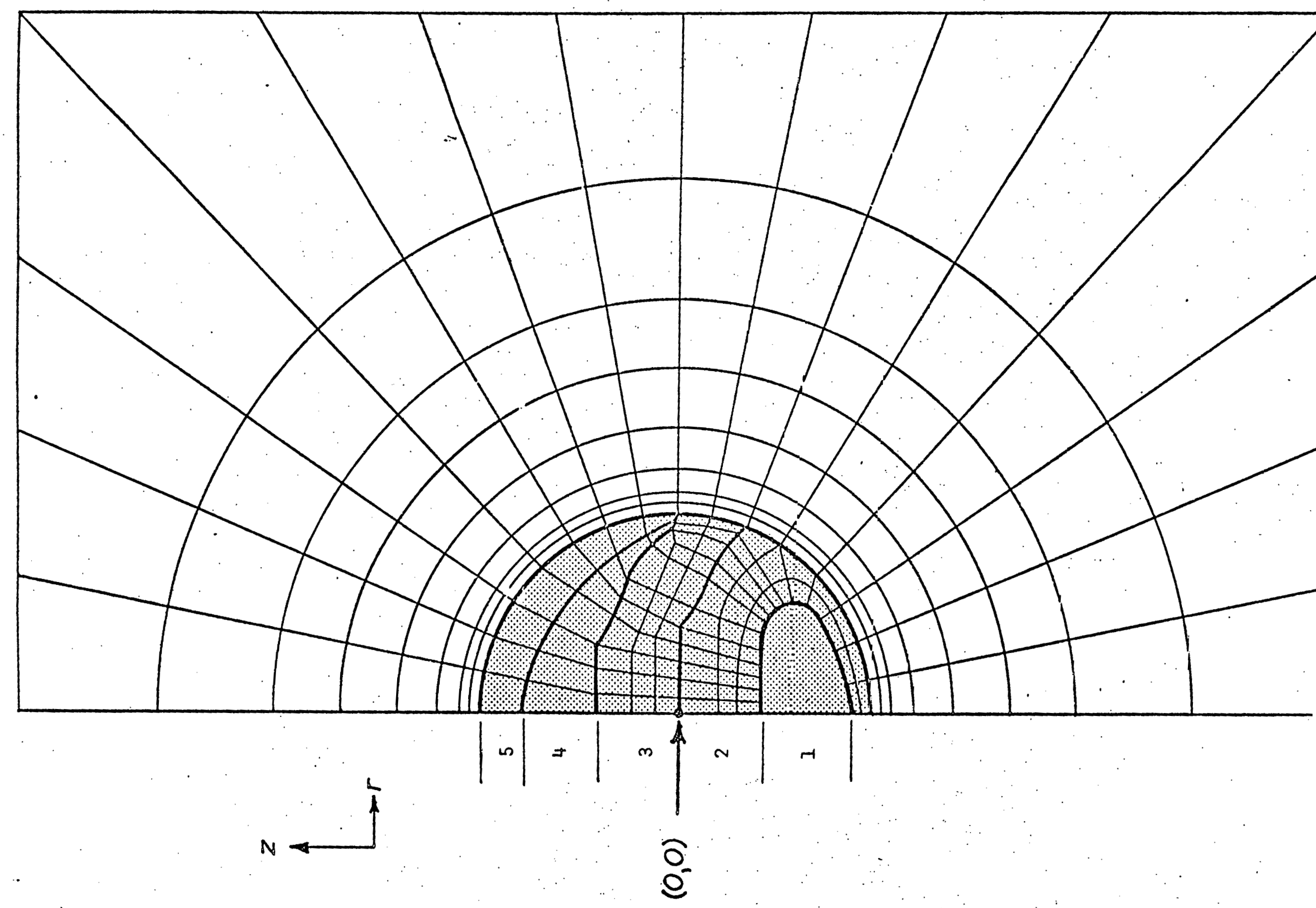




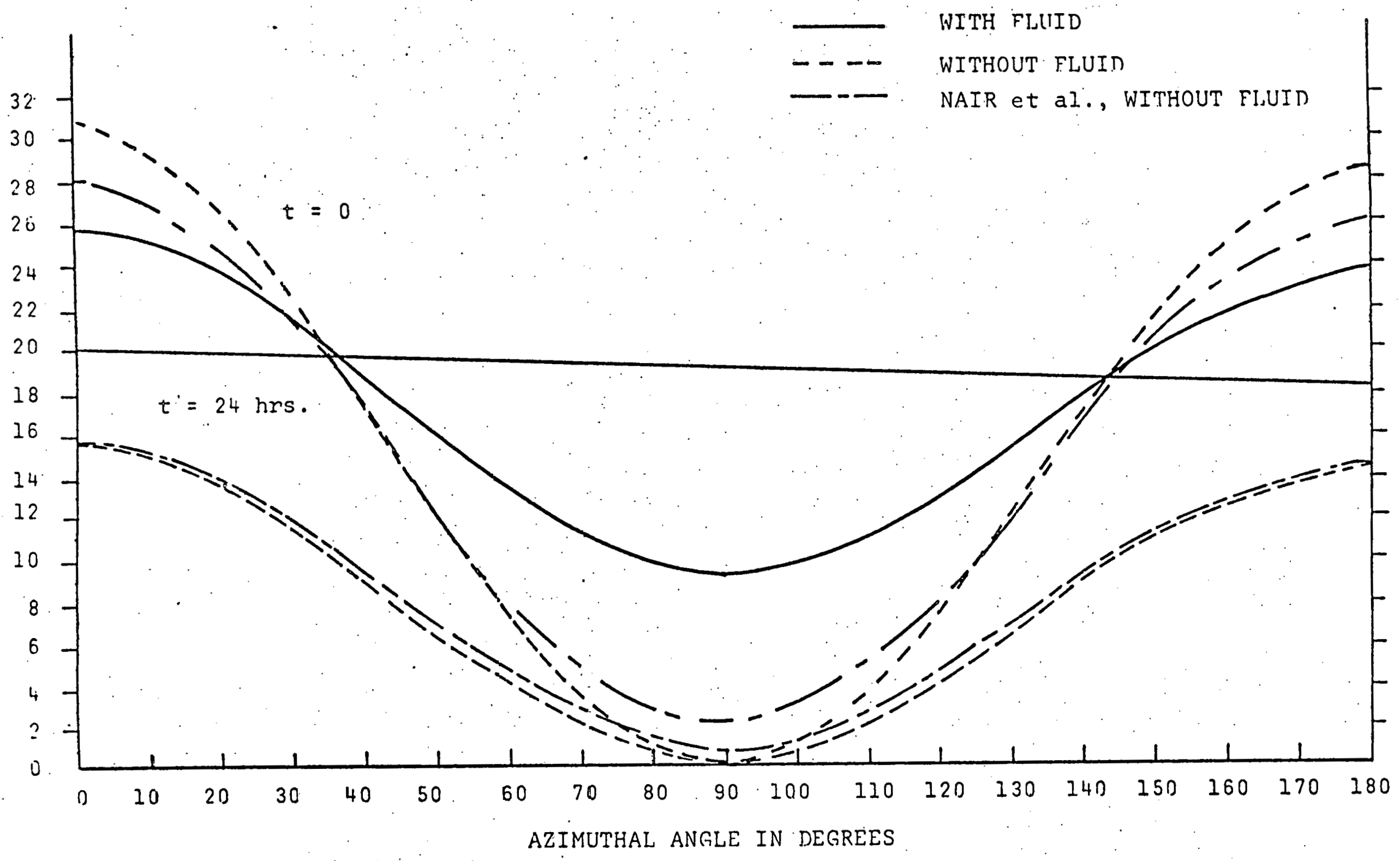

EIGURE 2 


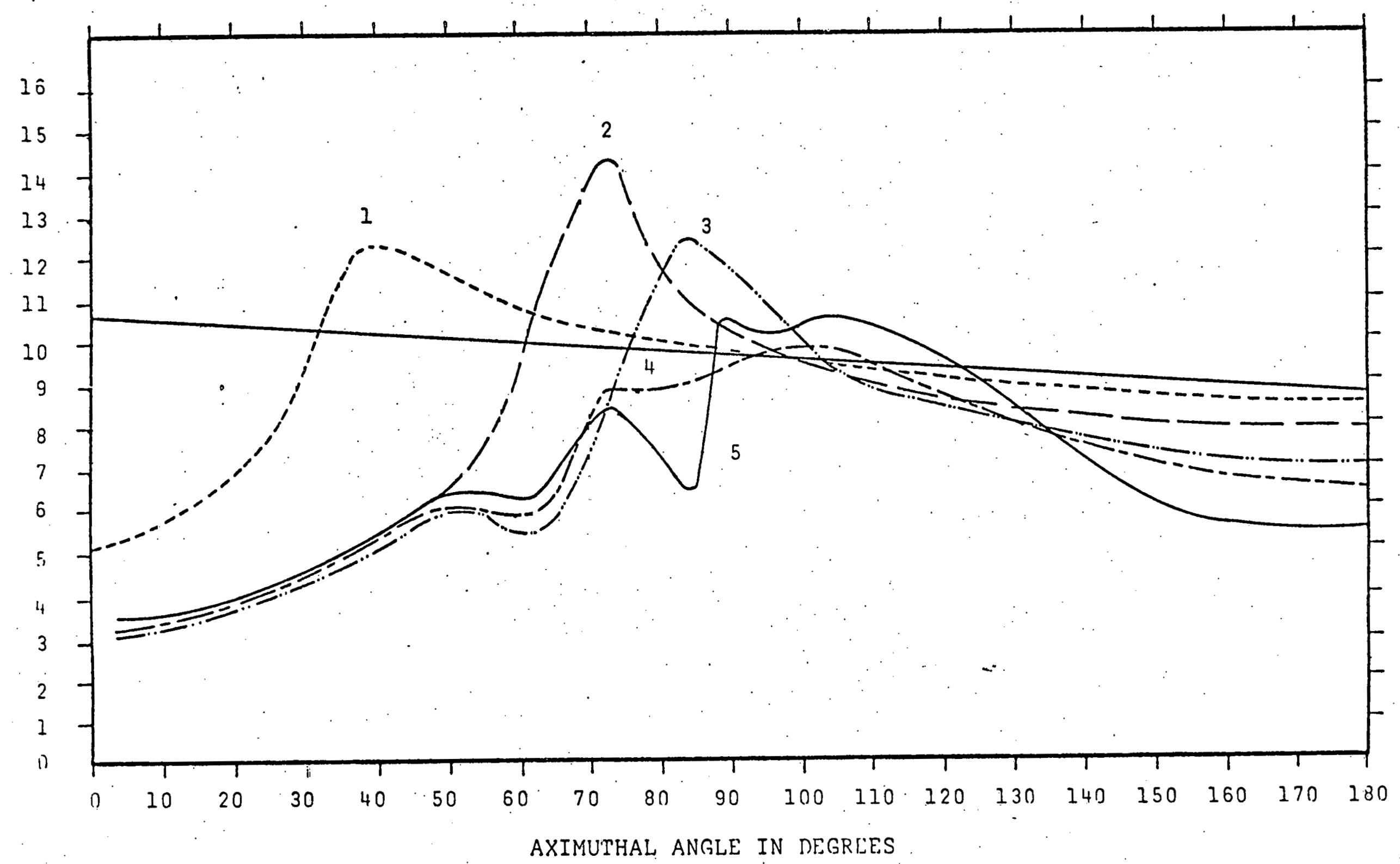

FIGURE 4 


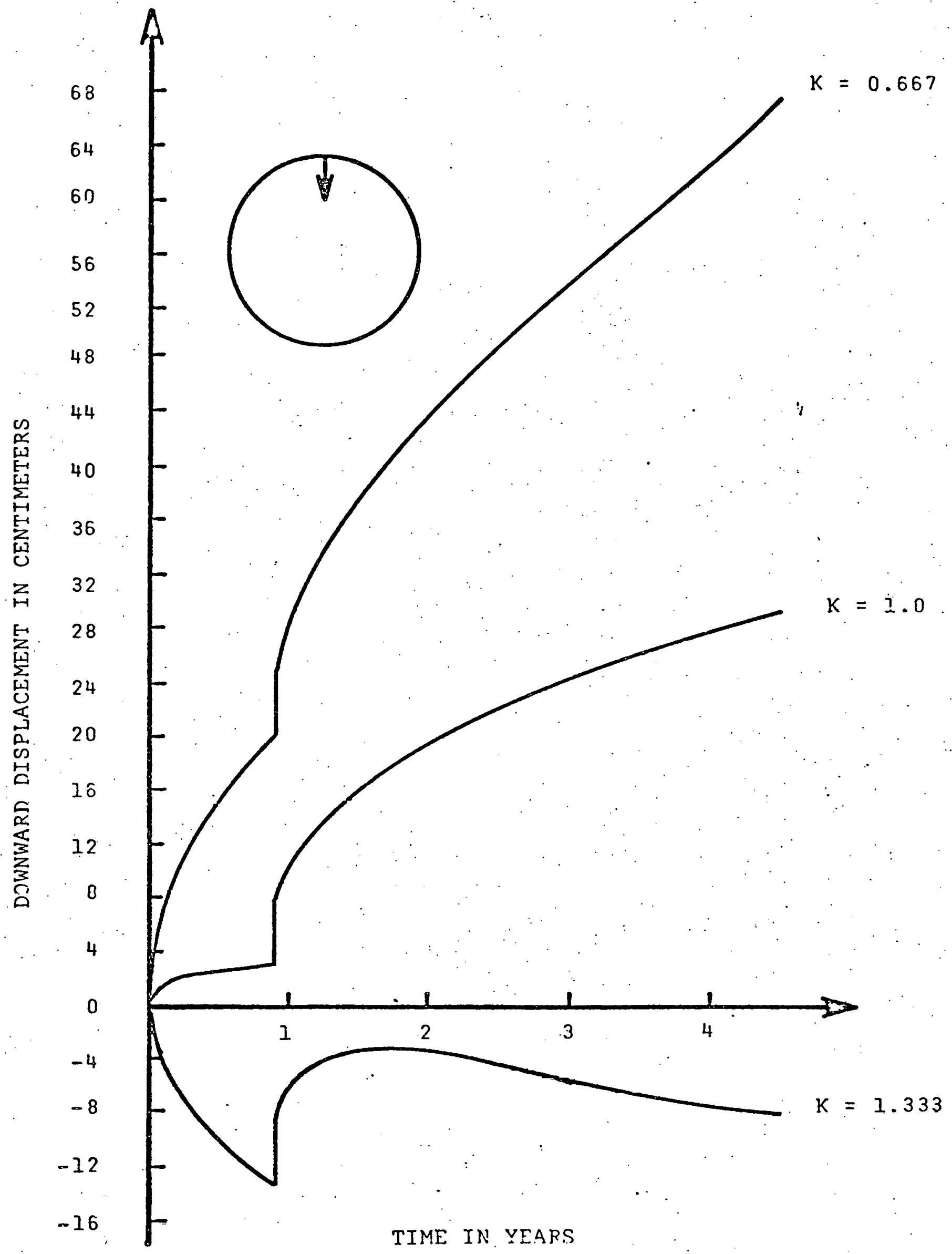

FIGURE 5 


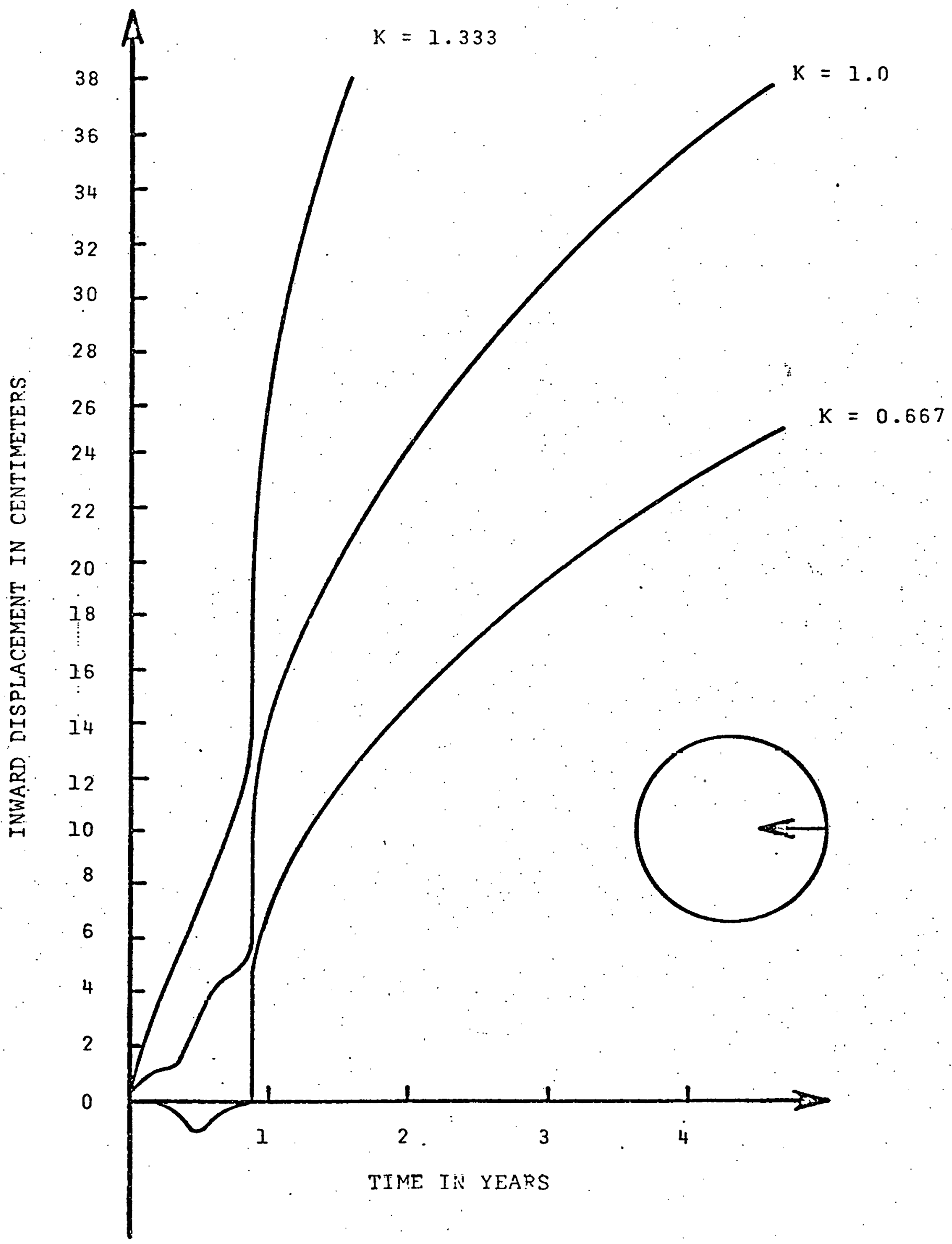

FIGURE 6 


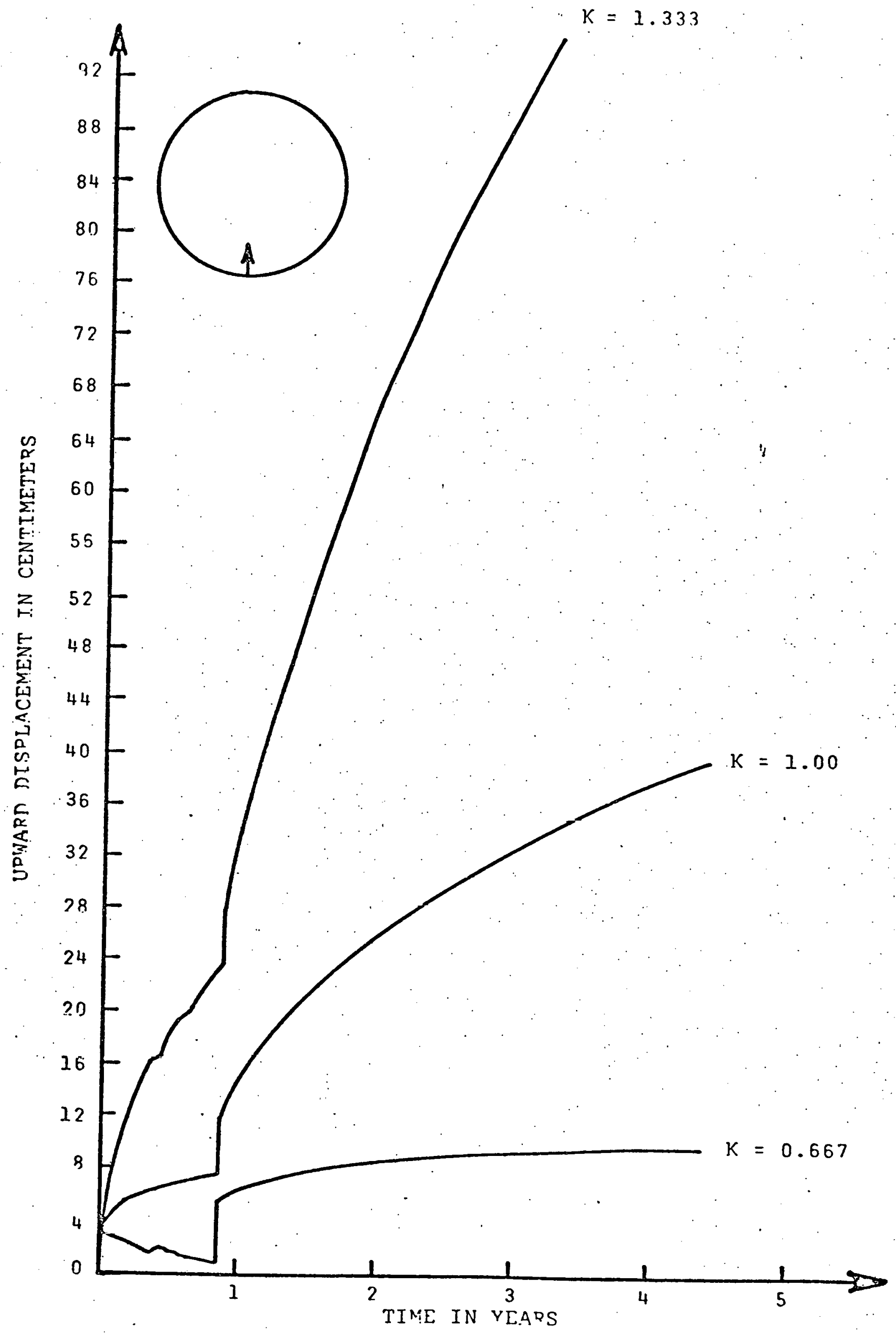

